\title{
Nonsurgical Management of Traumatic Internal Jugular Vein Rupture Using Direct Compression
}

\author{
Chan Yong Park', Wu Seong Kang', Sang Hyun $\mathrm{Seo}^{2}$, Sung Nam Moon ${ }^{2}$ \\ 'Department of Trauma Surgery, 'Department of Radiology, Wonkwang University, Jeonbuk, Korea
}

The internal jugular vein (IJV) very rarely gets ruptured by blunt trauma and is usually surgically repaired. Here, we report a case of successful nonsurgical treatment using external compression in patient with ruptured left IJV with multifocal active bleeding in neck computed tomography.

(Trauma Image Proced 2018(2):62-64)

Key Words: Internal jugular vein; Blunt trauma; Non-operative treatment; External compression; Active bleeding

\section{CASE}

A 67-year-old male presented to the emergency department with left neck pain and swelling after meeting with a traffic accident. The neck computed tomography (CT) revealed a multifocal contrast media extravasation from the left internal jugular vein (IJV) and a severe mass affecting the diffuse soft tissue infiltrating and thickening the deep cervical and superficial cervical spaces (Fig. 1). The left cervical portion was compressed with a sand bag (Fig. 2). The patency of both IJV was confirmed in the neck ultrasound on the following morning (Fig. 3). One week later, follow-up CT revealed total recanalization of the occluded left IJV, marked absorption of preexisting hematoma located in the deep neck spaces, and a bulging caused by liquefaction of the resolving hematoma in the left masticator and posterior cervical space (Fig. 4). The left IJV, not found in threedimensional angiographic images in the neck CT, was observed again after 1 week (Fig. 5).

\section{DISCUSSION}

Ruptured IJV due to trauma has been rarely reported (1-3). In particular, reports of rupture due to blunt trauma are very rare. Simmons et al. (2) reported that 3 of 5 isolated IJV injuries underwent primary repair and two underwent ligation. Yen et al. (3) reported a case treated by embolization for a penetrating IJV injury accompanied with life-threatening hemorrhage. Park et al. (4) reported endovascular repair following cardiopulmonary resuscitation in a patient with common iliac vein rupture caused by blunt trauma. However, only a few cases had successful nonsurgical management of ruptured IJV due to blunt trauma. External compression in patients with isolated IJV injury associated with multifocal contrast media extravasation after blunt trauma may be considered as a treatment option in selected patients.

Received: October 22, 2018 Revised: October 27, 2018 Accepted: October 29, 2018

Correspondence to: Chan Yong Park, Department of Trauma Surgery, Wonkwang University Hospital, Jeonbuk, Korea

Tel: 82-63-859-2602, Fax: 82-63-859-2029, E-mail: wkafyddl@hanmail.net

Copyright (c) 2018 Korean Association for Research, Procedures and Education on Trauma. All rights reserved.

@This is an open-access article distributed under the terms of the Creative Commons Attribution Non-Commercial License (http://creativecommons.org/ licenses/by-nc/4.0) which permits unrestricted noncommercial use, distribution, and reproduction in any medium, provided the original work is properly cited 


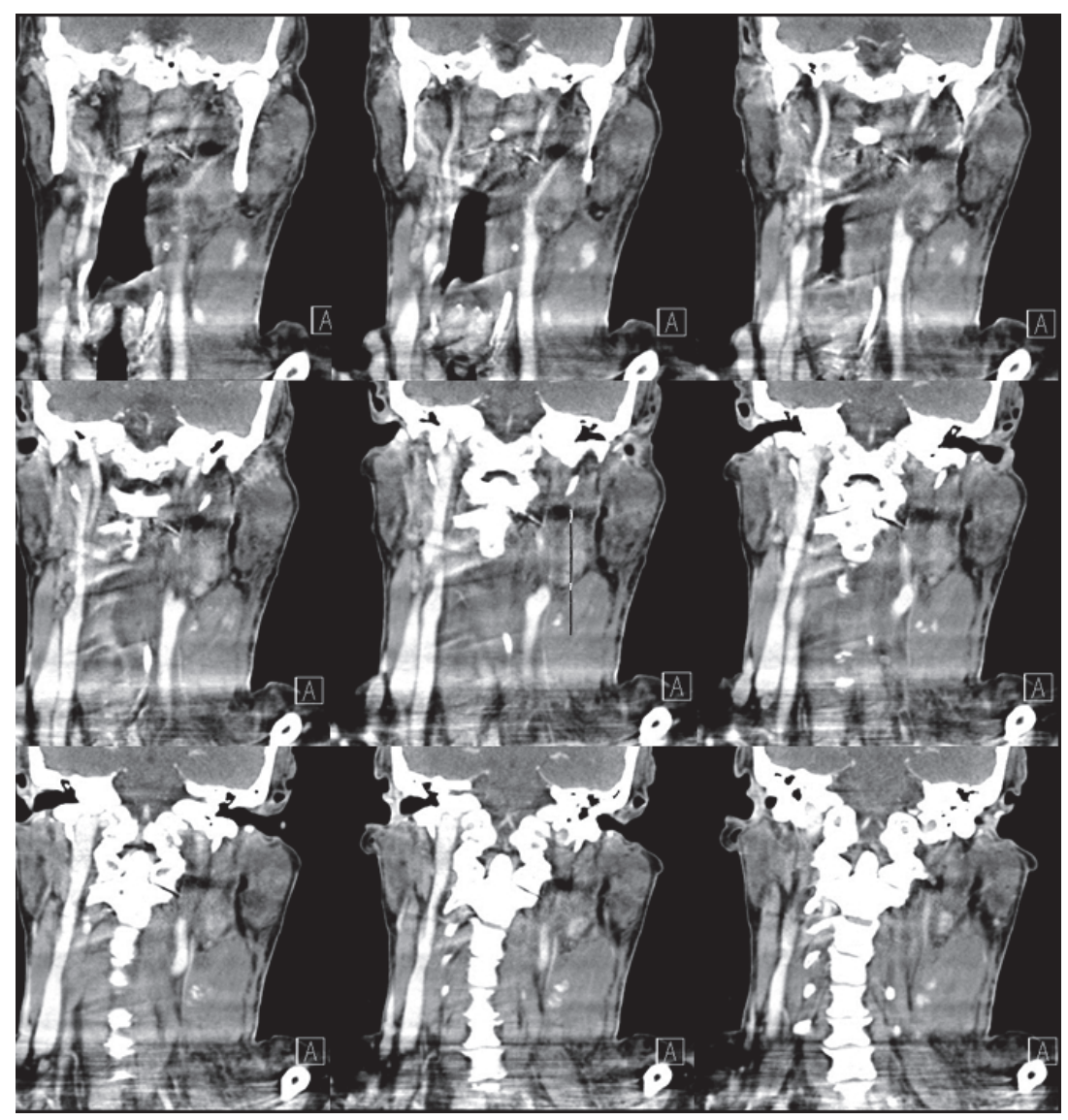

Fig. 1. Neck CT revealing multifocal contrast media extravasation accompanied with severe mass effect from the left internal jugular vein (IJV).

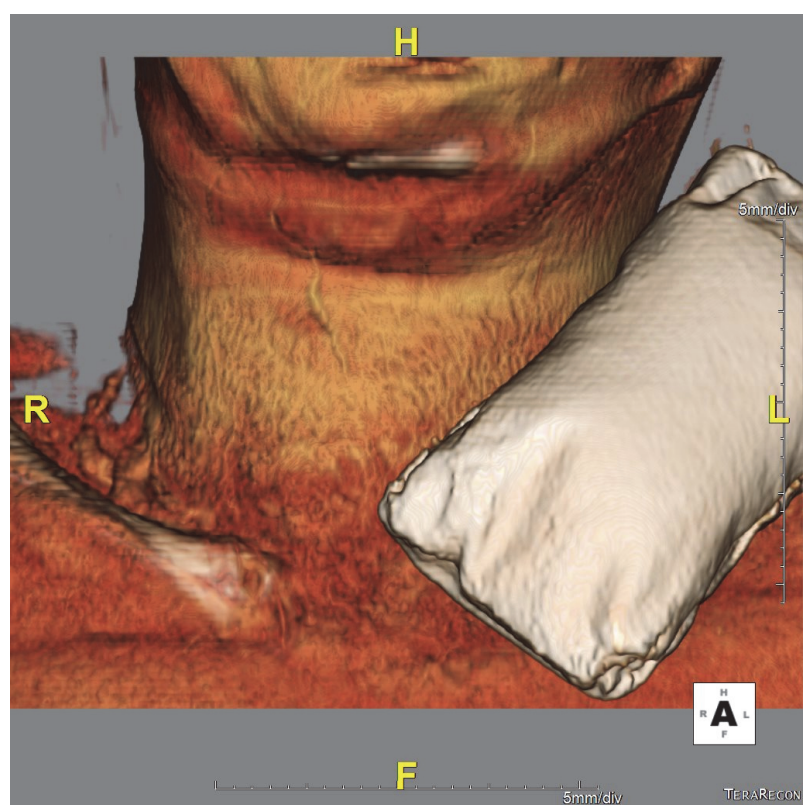

Fig. 2. The left cervical portion is compressed with a sand bag.

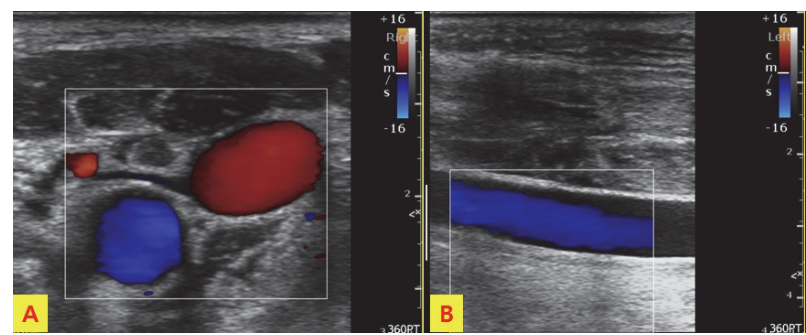

Fig. 3. The patency of the left internal jugular vein (IJV) is observed in the neck ultrasound on the following morning. Flows in the left common carotid artery (indicated by blue color) and left IJV (indicated by red color) are observed in the short axis view (A). In the long axis view, a flow in the left IJV (indicated by blue color) is observed. 


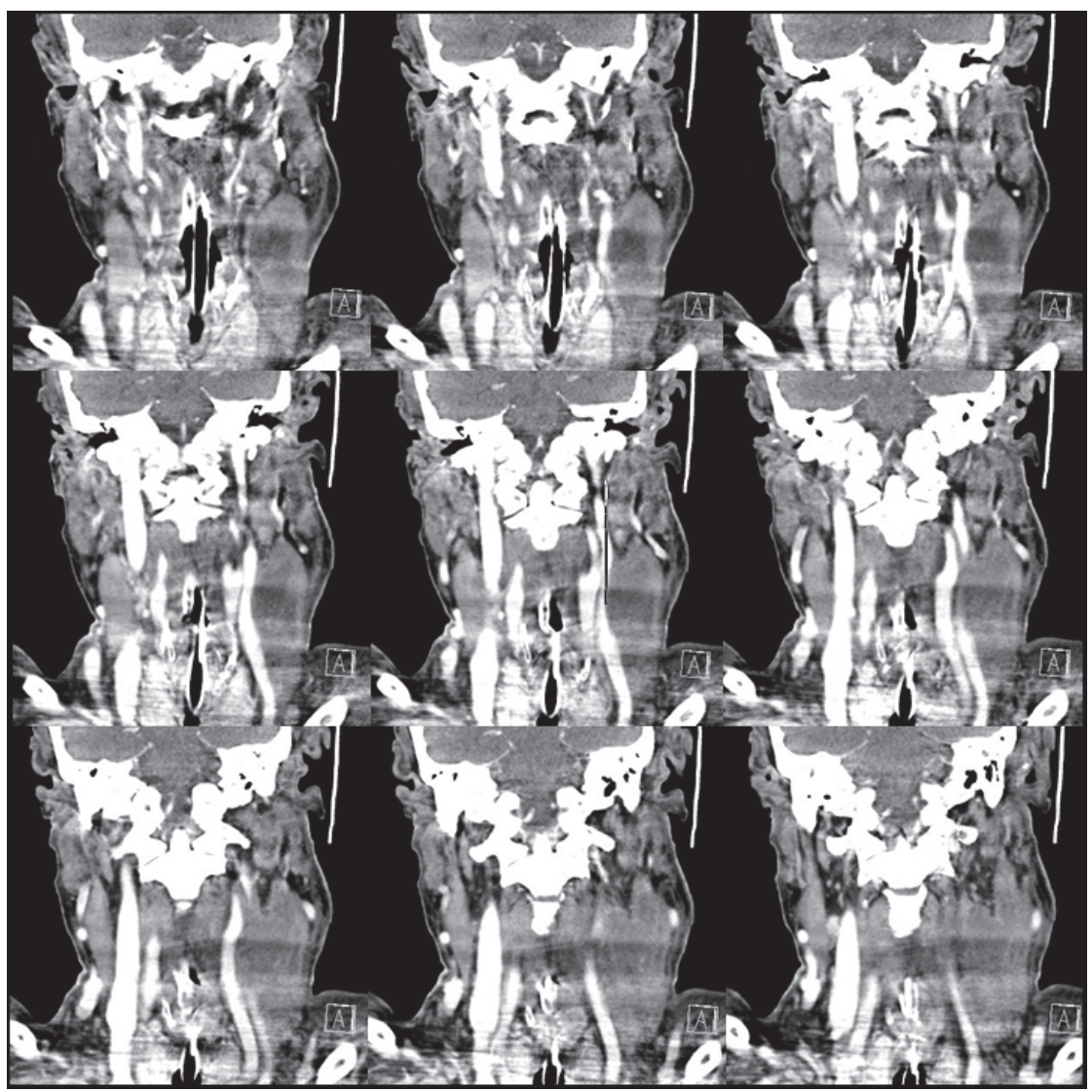

Fig. 4. One week later, follow-up CT reveals total recanalization of the occluded left internal jugular vein (IJV) and marked absorption in the deep neck hematoma.

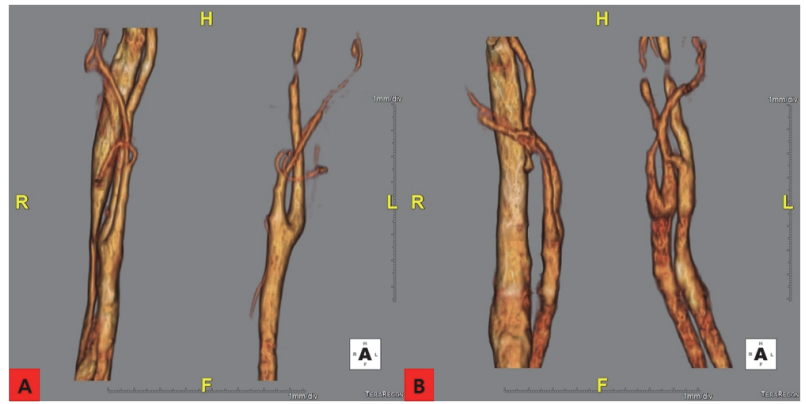

Fig. 5. Neck CT (3-D angiographic images) shows a remarkable change in the left internal jugular vein (IJV), which was not observed upon admission (A). After 1 week, the left IJV is clearly observed (B).

\section{Conflict of Interest Statement}

None of authors have a conflict of interest

\section{REFERENCE}

1. Clark JD. Blunt neck trauma: torn internal jugular vein after a motor vehicle crash. J Emerg Nurs. 2008;34(3): 249-50.

2. Simmons JD, Ahmed N, Donnellan KA, Schmieg RE Jr, Porter JM, Mitchell ME. Management of traumatic vascular injuries to the neck: a 7-year experience at a Level I trauma center. Am Surg. 2012;78(3):335-8.

3. Yen AJ, Conrad MB, Loftus PA, Kumar V, Nanavati SM, Wilson MVV, et al. Internal Jugular Vein Embolization to Control Life-Threatening Hemorrhage after Penetrating Neck Trauma. J Vasc Interv Radiol. 2018;29(3):435-7.

4. Park CY, Yeo KH, Kim HH, Hwang JJ, Lee SC, Kim CW, et al. Endovascular Repair of Traumatic Common Iliac Vein Rupture. Trauma Image Proced. 2016;1(1):26-8. 